\title{
QT dispersion is not related to infarct size or inducibility in patients with coronary artery disease and life threatening ventricular arrhythmias
}

\author{
J De Sutter, R Tavernier, C Van de Wiele, J De Backer, J Kazmierczak, G De Backer, \\ R Dierckx, L Jordaens
}

\begin{abstract}
Objective-To relate QT parameters to infarct size and inducibility during electrophysiological studies.

Design-Analysis of a prospective register. Setting-University hospital.

Patients-64 patients with coronary artery disease and documented life threatening ventricular arrhythmias.

Interventions-Measurements of QTmax, QTc-max, and QT dispersion (QT-d) on a simultaneous 12 lead ECG (50 $\mathrm{mm} / \mathrm{s}$ ). Estimation of myocardial infarct size with radionuclide left ventricular ejection fraction (LVEF), echocardiography (left ventricular end diastolic diameter, LVEDD), and a defect score based on a quantitative stress redistribution 201-thallium perfusion study. Electrophysiological study to assess inducibility. Results-Mean (SD) QT parameters were: QT-max 440 (50) ms, QTc-max 475 (46) ms, and QT-d 47 (20) ms. Mean (SD) estimates of infarct size were: LVEF 34 (13)\%, LVEDD 61 (9) $\mathrm{mm}$, and defect score 18 (11). There was no significant correlation between any index of infarct size and QT parameters. QT parameters were not significantly different between patients with inducible $(n=57)$ and noninducible arrhythmias $(\mathbf{n}=7)$ (QT-max: 416 (30) $v 443$ (51) ms, $p=0.18$; QTc-max 485 (34) $v 473$ (47) ms, p = 0.34; QT-d 47 (12) $v 47(21) \mathrm{ms}, \mathrm{p}=\mathbf{0 . 7 3})$. Non-inducible patients had a significant lower defect score: $8(9) v 19(11), p=0.02$, but comparable LVEF: $38(12) \% v 34(12) \%, p=0.58$, and LVEDD: $54(10) v 61(8) \mathrm{mm}, \mathrm{p}=0.13$. Conclusions-QT parameters are not influenced by infarct size and do not predict inducibility during electrophysiological study in patients with coronary artery disease and malignant ventricular arrhythmias. In contrast, the amount of scar tissue determined by perfusion imaging is strongly correlated with inducibility.

(Heart 1999;81:533-538)
\end{abstract}

Keywords: QT parameters; infarct size; electrophysiological testing; perfusion imaging

QT dispersion (that is, the difference between the maximum and the minimum QT interval measured on the 12 lead ECG) has been suggested as a non-invasive index of regional ventricular repolarisation inhomogeneity. An abnormally increased QT dispersion has been observed in patients with acute myocardial infarction, ${ }^{1}$ heart failure, and sudden death ${ }^{2}{ }^{3}$ and has been associated with an increased risk of ventricular tachyarrhythmias. ${ }^{4-6}$

Myocardial infarction profoundly influences the depolarisation and therefore the repolarisation sequence of the ventricles. A relation between infarct size and QT dispersion is thus expected, but this has not been studied in detail. Furthermore, there are few data relating inducibility of ventricular tachycardia to QT parameters in patients with coronary artery disease, that would support the routine use of the former in clinical practice. ${ }^{7}$

Our aim in this study was to assess the relation between QT parameters and measurements of infarct size, and the relation between QT parameters and inducibility of sustained ventricular tachycardia or ventricular fibrillation during electrophysiological testing, in patients with coronary artery disease and ventricular tachycardia or aborted sudden cardiac death.

\section{Methods}

STUDY POPULATION

Between January 1995 and September 1997, we examined 102 consecutive patients admitted to the University Hospital Gent, Belgium, for diagnostic work up and treatment of ventricular tachycardia or aborted sudden death. On the basis of medical history, 12 lead resting ECG, and coronary angiography, 64 patients were considered to have coronary artery disease complicated by ventricular tachycardia $(V T)(n=46)$ or ventricular fibrillation (VF) $(n=18)$ and formed the study population.

From the clinical history, the presence of $\mathrm{Q}$ waves on the resting ECG, or the presence of occlusion of one of the three major coronary arteries, 54 patients had sustained a myocardial infarct. Their infarcts were localised in the inferior region (group A, Q waves in II, III, and $\mathrm{aVF}$, or occlusion of the right coronary artery, $\mathrm{n}=37$ ), or the anterior region (group $\mathrm{B}, \mathrm{Q}$ waves in the precordial leads, or occlusion of the circumflex artery or left anterior descending coronary artery, $\mathrm{n}=17$ )

Patients without $\mathrm{Q}$ waves and no complete occlusion of one of the coronary arteries, but with one or more significant stenosis $(>75 \%)$, were considered to have coronary artery disease without clear evidence of previous myocardial infarction (group $\mathrm{C}, \mathrm{n}=10$ ). 
In all patients QT measurements, electrophysiological study results, and measurements of infarct size (radionuclide left ventricular ejection fraction, 201-thallium myocardial perfusion imaging, and resting echocardiography) were obtained within 14 days of admission.

QT MEASUREMENTS

A simultaneous 12 lead ECG taken on the day of the electrophysiological study with a paper speed of $50 \mathrm{~mm} / \mathrm{s}$ was used for QT measurements. The QT interval was taken as the interval from the onset of the QRS complex to the end of the $T$ wave, defined as the intersection of the isoelectric line and the $\mathrm{T}$ wave. In the presence of a $U$ wave, the end of the QT interval was taken to be the nadir between the $\mathrm{T}$ and $\mathrm{U}$ wave peaks. No extrasystolic or postextrasystolic QT intervals were included. ${ }^{5-10}$ The maximum QT interval was corrected for heart rate using Bazett's formula (QTc-max). ${ }^{11}$ For QT dispersion (QT-d), the difference between the minimum and the maximum QT interval on the 12 lead ECG was used if at least eight leads were suitable for analysis. Measurements were done by two investigators blinded to the result of the other studies, and mean values were calculated. Four patients with ventricular pacing were excluded.

MEASUREMENTS OF INFARCT SIZE

201-Thallium myocardial perfusion imaging

Bicycle stress or dipyridamole stress thallium tomography was performed in prone position using a triple headed Toshiba gamma camera (GCA 900 A, Toshiba, Tokyo, Japan) following injection of $111 \mathrm{MBq}$ upon approaching predefined end points: severe angina, $>2 \mathrm{~mm}$ ST segment displacement, hypotension or sustained tachyarrhythmias, physical exhaustion, or severe dyspnoea. The camera was rotated in $6^{\circ}$ increments, collecting views over $360^{\circ}$ for 30 seconds each, using an elliptical orbit. Matrix size was $64 \times 64$. Rest studies were acquired using the same imaging protocol four hours later. A 17 segment, five point score $(0=$ normal to $4=$ severely reduced tracer uptake) was used for the semiquantitative analysis of the images. Each segment with reduced uptake during stress (score more than 0 ), which did not change during redistribution, was considered to have a fixed defect. Its severity was defined by the point score. A total defect score was calculated by summation of the individual scores of each fixed defect. Segments showing an improvement in perfusion during redistribution were defined as ischaemic. Segments showing worse perfusion during redistribution compared with the stress images were defined as showing reverse redistribution. All images were scored by two independent investigators who were blinded to the results of the electrophysiological study. We obtained $\kappa$ values of 0.92 for the interobserver agreement and 0.96 for the intraobserver agreement. Differences were resolved by consensus.
Radionuclide left ventricular ejection fraction Radionuclide equilibrium angiographic data were acquired in left anterior oblique view on a small field of view Toshiba triple headed gamma camera, equipped with low energy high resolution collimators. Images with approximately six million counts were obtained using 16 frames with a pixel size of $3.4 \mathrm{~mm}$. Cardiac cycles with RR intervals not within $10 \%$ of the average were rejected. Left ventricular ejection fractions (LVEF, \%) were determined by means of a commercially available soft ware algorithm provided by Toshiba.

Resting echocardiography

Resting echocardiographic examination was performed with the patient in the semilateral position. Cross sectional imaging in the left parasternal long axis plane was used to guide M mode recordings of the left ventricular minor axis, with the cursor by the tips of the mitral leaflets. Left ventricular end diastolic dimensions (LVEDD, mm) was measured on the minor axis $M$ mode recording from the leading edge of the septal endocardium to that of the posterior wall, at the onset of the $\mathrm{Q}$ wave of the ECG.

\section{ELECTROPHYSIOLOGICAL STUDY}

All patients underwent an electrophysiological study after an overnight fast and after mild sedation with $5 \mathrm{mg}$ of diazepam. Three quadripolar catheters were inserted percutaneously and positioned in the high right atrium, across the tricuspid valve for $H$ is bundle recording, and at the right ventricular apex. Stimulation in the ventricle was delivered at twice diastolic threshold. The stimulation protocol consisted of a basic train of eight impulses (600 ms, $460 \mathrm{~ms}$, and $400 \mathrm{~ms}$ ) and up to four extrastimuli. Stimulation was performed in the right ventricular apex and the right ventricular outflow tract. Sustained VT was defined as a monomorphic VT lasting $\geqslant 30$ seconds or as VT with haemodynamic compromise requiring termination. A negative study was defined by the inability to induce sustained VT or VF. ${ }^{12}$

STATISTICAL ANALYSIS

For statistical analysis we used the SPSS for Windows package release 7.5. All data are expressed as mean (SD) or median (range). Spearman correlation coefficients were used in determining univariate correlations between QT parameters and measures of infarct size. Comparison of values among subgroups for localisation of myocardial infarction was done by one way analysis of variance (ANOVA) and the Student-Newman-Keuls test for multiple ranks. A Mann-Whitney U test was used to compare the inducible and the non-inducible group. A probability ( $p$ ) value of $<0.05$ was considered statistically significant.

\section{Results}

CLINICAL CHARACTERISTICS

The mean (SD) age of the 64 study patients was 66 (9) years and 61 (95\%) were male. A clinical history of myocardial infarction was present in 54 patients $(84 \%)$. The mean time 
Table 1 Correlations between $Q T$ parameters and measurements of infarct size $(n=40)$

\begin{tabular}{llll}
\hline & 201-Thallium defect score & Radionuclide LVEF & Echocardiographic LVEDD \\
\hline QT-d & $r=0.25(\mathrm{p}=0.20)$ & $r=-0.07(\mathrm{p}=0.68)$ & $r=-0.01(\mathrm{p}=0.96)$ \\
QT-max & $r=0.16(\mathrm{p}=0.42)$ & $r=0.04(\mathrm{p}=0.83)$ & $r=0.06(\mathrm{p}=0.72)$ \\
QTc-max & $r=0.05(\mathrm{p}=0.81)$ & $r=-0.11(\mathrm{p}=0.50)$ & $r=-0.08(\mathrm{p}=0.66)$ \\
\hline
\end{tabular}

LVEDD, left ventricular end diastolic diameter; LVEF, left ventricular ejection fraction; QT-d, QT dispersion; QT-max, maximum QT interval; QTc-max, corrected maximum QT interval.

between the myocardial infarction and the episode of VT or VF was 134 (113) months (range 1 to 432 months). Nine patients had their infarcts within the previous six months. Nineteen patients $(30 \%)$ had previous coronary artery bypass grafting or coronary angioplasty. The clinical presentation on admission was haemodynamically poorly tolerated VT $(72 \%)$ or VF $(28 \%)$.

Coronary risk factors were hypertension in 21 patients (33\%), smoking in $44(69 \%)$, hypercholesterolaemia in $29(45 \%)$, and diabetes in $10(16 \%)$.

All patients underwent coronary angiography which showed one vessel disease in 13 $(20 \%)$, two vessel disease in $19(30 \%)$, and three vessel disease in $32(50 \%)$. At the time of the electrophysiological study, $47 \%$ of the patients were on $\beta$ blockers, $86 \%$ on angiotensin converting enzyme inhibitors, $75 \%$ on aspirin, and $41 \%$ on nitrates. All antiarrhythmic drugs were stopped two days before the electrophysiological study.

RESULTS OF THE ELECTROPHYSIOLOGICAL STUDY Forty four patients $(69 \%)$ had inducible sustained monomorphic VT and 13 (20\%) had inducible VF during the electrophysiological study. These two groups of patients $(n=57$, $89 \%$ ) were predefined as inducible. Nonsustained VT could be induced in two patients $(5 \%)$, and in five patients ( $8 \%)$ no arrhythmia could be induced. These two groups of patients were predefined as non-inducible $(n=7$, $11 \%)$. There was no significant difference in age between the inducible and non-inducible patients (66 (9) years $v 61$ (9) years, $\mathrm{p}=0.19$ ).

QT PARAMETERS AND MEASUREMENTS OF INFARCT SIZE

Of the 64 study patients, 40 (63\%) were included for QT measurements. Four patients $(6 \%)$ were excluded because of a ventricular pacemaker. In 20 patients (31\%), the end of the $\mathrm{T}$ wave could not be identified reliably in four or more leads and these patients were therefore excluded. Among the 40 patients

Table 2 QT parameters, 201-thallium defect score, and radionuclide left ventricular ejection fraction (LVEF) in the three study groups

\begin{tabular}{lllll}
\hline & $\begin{array}{l}\text { Group } A \\
(n=37)\end{array}$ & $\begin{array}{l}\text { Group } B \\
(n=17)\end{array}$ & $\begin{array}{l}\text { Group } C \\
(n=10)\end{array}$ & $\begin{array}{l}\text { p Value } \\
\text { (one way ANOVA) }\end{array}$ \\
\hline QT-d (mm) & $50(23)$ & $45(18)$ & $38(13)$ & 0.58 \\
QT-max (mm) & $435(50)$ & $423(46)$ & $424(54)$ & 0.17 \\
Qtc-max (mm) & $472(45)$ & $472(39)$ & $496(68)$ & 0.73 \\
Tl defect score & $20(10)$ & $18(12)$ & $7(10)^{\star}$ & 0.03 \\
LVEF (\%) & $36(12)$ & $26(11) \dagger$ & $39(17)$ & 0.02 \\
\hline
\end{tabular}

Values are mean (SD).

${ }^{\star} \mathrm{p}<0.05$, group $\mathrm{C} v$ group $\mathrm{A}$ and group $\mathrm{C} v$ group $\mathrm{B} ; \mathrm{t} \mathrm{p}<0.05$, group $\mathrm{B} v$ group $\mathrm{A}$ and group $\mathrm{B} v$ group C.

ANOVA, analysis of variance; group A, previous inferior infarct; group B, previous anterior infarct; group C, coronary artery disease without ECG or angiographic evidence of myocardial infarction; QT-d, QT dispersion; QT-max, maximum QT interval; QTc-max, corrected maximum QT interval.

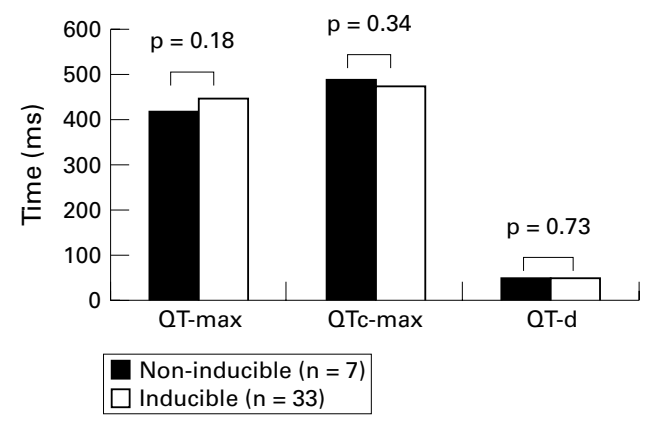

Figure 1 QT parameters in patients with non-inducible $(n=7)$ and inducible $(n=33)$ arrhythmias. There were no significant differences in any $Q T$ parameter between the two groups. QT-d, QT dispersion; $Q T-$ max, maximum $Q T$ interval; $Q T$ T-max, corrected maximum $Q T$ interval.

finally included, interobserver variability was within $11 \mathrm{~ms}$ for the measurement of QT dispersion. The correlation coefficient for QT dispersion measurements between both observers was $0.77(\mathrm{p}<0.05)$. Mean QT dispersion was 47 (20) ms, QT-max 440 (50) ms, and QTc-max 475 (46) ms.

The following direct and indirect measurements of infarct size were obtained: 201thallium perfusion defect score 18 (11), radionuclide LVEF 34 (13)\%, and LVEDD 61 (9) $\mathrm{mm}$. The correlations between QT parameters and measures of infarct size are summarized in table 1. No significant correlation was found between any QT parameter and any index of myocardial infarct size.

QT parameters, thallium defect scores, and radionuclide LVEF for the different subgroups (group A: previous inferior myocardial infarct; group B: previous anterior myocardial infarct; and group C: no clear arguments for previous myocardial infarct) are shown in table 2 . Although the thallium defect score was significantly lower in group C ( $v$ group A and group $\mathrm{B})$ and the radionuclide LVEF was significantly lower in group B ( $v$ group A and group C), no significant differences were found for the QT parameters between the different groups.

QT PARAMETERS AND MEASUREMENTS OF INFARCT SIZE AND INDUCIBILITY DURING ELECTROPHYSIOLOGICAL STUDY

QT parameters were available for all seven non-inducible patients and for 33 of the inducible patients. Patients who were not included for QT analysis had no significant differences in clinical characteristics or measures of infarct size compared with the patients who were included. As shown in fig 1 , no significant differences were found between the non-inducible and the inducible group for QT-max (416 (30) $v 443$ (51) ms, $\mathrm{p}=0.18$ ), QTc-max (485 (34) $v 473$ (47) $\mathrm{ms}, \mathrm{p}=0.34$ ), and QT-d (47 (12) v 47 (21) ms, p = 0.73).

Table 3 shows the differences between the measurements of infarct size for the noninducible and inducible groups. On 201thallium perfusion imaging, the non-inducible patients had significantly fewer segments with fixed defects (3 (3) $v 7(4), \mathrm{p}=0.01)$ and a significantly lower defect score (8 (9) $v 19$ (11), $\mathrm{p}=0.02)$. Radionuclide LVEF and 
Table 3 Results of 201-thallium perfusion imaging, left ventricular ejection fraction (LVEF), and echocardiographic left ventricular end diastolic diameter (LVEDD) in patients with and without inducible ventricular tachycardia or fibrillation

\begin{tabular}{|c|c|c|c|}
\hline & $\begin{array}{l}\text { Inducible } \\
(n=57)\end{array}$ & $\begin{array}{l}\text { Non-inducible } \\
(n=7)\end{array}$ & p Value \\
\hline \multicolumn{4}{|c|}{ 201-thallium perfusion imaging } \\
\hline Fixed defects & $7(4)$ & $3(3)$ & 0.01 \\
\hline Ischaemic defects & $2(3)$ & $4(4)$ & 0.16 \\
\hline Normal perfusion & $7(3)$ & $10(4)$ & 0.06 \\
\hline Reverse redistribution & $1(1)$ & $0(0)$ & 0.18 \\
\hline Defect score & $19(11)$ & $8(9)$ & 0.02 \\
\hline Radionuclide LVEF (\%) & $34(12)$ & $38(12)$ & 0.58 \\
\hline LVEDD $(\mathrm{mm})$ & $61(8)$ & $54(10)$ & 0.13 \\
\hline
\end{tabular}

Values are mean $(\mathrm{SD})$

echocardiographic LVEDD measurements were not significantly different between the groups.

\section{Discussion}

The principal findings of this study are that QT parameters (QT-d, QT-max, and QTc-max) in patients with coronary artery disease and life threatening ventricular arrhythmias are not determined by the degree of left ventricular dysfunction, left ventricular dilatation, or amount of scar tissue evaluated by myocardial perfusion imaging. Furthermore, QT parameters did not predict arrhythmia inducibility during electrophysiological study in these patients, while the amount of scar tissue on perfusion imaging was strongly related to inducibility.

QT PARAMETERS AND MEASUREMENTS OF INFARCT SIZE

Increased QT dispersion values have been associated with an increased risk of ventricular arrhythmias $^{5613}$ and sudden cardiac death. ${ }^{3}$ The explanation for this association is, however, unclear. It has been suggested that the increased QT dispersion could reflect the degree of left ventricular dysfunction, and be an index of left ventricular damage. However in our present study, as in two previous ones, ${ }^{614}$ no significant correlation was found between ejection fraction and QT dispersion in patients with coronary artery disease and myocardial infarction. Also, despite the fact that patients with anterior myocardial infarction (group B) had a significantly lower LVEF than the patients in group A and C, QT parameters were not significantly different. Only Pye et al found a significant correlation between QT dispersion and LVEF. ${ }^{5}$ In that study, however, a mixed population of patients with coronary artery disease, dilated cardiomyopathy, and some without evidence of heart disease was evaluated.

A second explanation for an increase of QT dispersion could be the presence of larger amounts of fibrous tissue, which may influence the homogeneity of repolarisation. ${ }^{15}$ However, we found no correlation between any QT parameter and the amount of scar tissue evaluated by thallium myocardial perfusion imaging. Furthermore, although the thallium defect score-as a measurement of scar tissue-was significantly lower in the group without clear evidence of a previous myocardial infarct (group C) compared with the groups with evidence of previous infarction (groups A and B), no significant differences were found for QT parameters between the groups.

Finally, dilatation of the infarcted ventricle may be accompanied by an increase of repolarisation inhomogeneity ${ }^{16}{ }^{17}$ and may lead to higher QT dispersion values. However, we found no significant correlation between the degree of dilatation measured by echocardiography and the QT dispersion values.

Taken together, our results suggest that QT dispersion in patients with coronary artery disease is not determined by the degree of left ventricular dysfunction, the amount of scar tissue, or the degree of left ventricular dilatation. Schneider et al recently suggested that QT dispersion is determined by the amount of viable myocardium in patients with chronic $Q$ wave myocardial infarction and mildly depressed left ventricular function. ${ }^{14}$ Because it is not clear whether their results are also applicable to other patient populations (for example, patients with more severely depressed left ventricular function, as in our study), further studies are needed to evaluate this possible explanation for an increase in QT dispersion.

QT PARAMETERS, MYOCARDIAL PERFUSION IMAGING, AND INDUCIBILITY DURING ELECTROPHYSIOLOGICAL STUDY

In this study, QT dispersion did not predict inducibility during electrophysiological study in patients with coronary artery disease and reduced left ventricular function. These results are comparable to those of Dobran et al, ${ }^{18}$ who found no difference in QT dispersion values between inducible and non-inducible patients with coronary artery disease and good left ventricular function. In contrast, Lee et al found that precordial QTapex dispersion was a predictor of inducible ventricular tachycardia. ${ }^{7}$ However, the predictive ability of QTapex dispersion was weak (a specificity of $75 \%$ and a sensitivity of $45 \%$ ) and heavily dependent on the inclusion of precordial lead V6. Furthermore, their patient population was inhomogeneous and consisted of patients with and without coronary artery disease. In our study, on the other hand, the amount of scar tissue determined by 201-thallium perfusion imaging was a strong predictor of inducibility. These results are in agreement with those of Gradel et $a l,{ }^{19}$ who recently showed that the size of scar, quantified by myocardial perfusion imaging, correlated well (and better than global left ventricular function) with inducibility of sustained VT in patients with coronary artery disease. Also, McFarland et al qualitatively compared perfusion imaging results with mapping of ventricular tachycardia and found that the site of origin of the tachycardia was located at the border of regions with fixed perfusion defects. ${ }^{20}$ These results and our own findings provide further evidence that the substrate for induction of VT or VF during electrophysiological study is myocardial scar tissue. Gioia et al recently showed that myocardial infarct scar 
size, as determined by SPECT imaging, was the most important predictor of survival in patients with coronary artery disease, impaired LVEF, and life threatening ventricular arrhythmias treated with implantable cardioverterdefibrillators (ICD). ${ }^{21}$ Preliminary results of our group ${ }^{22}$ further show that in these patients, myocardial infarct size determined on SPECT images is an independent predictor of appropriate shocks for recurrences of VT or VF. Taken together, these results suggest that quantitative analysis of myocardial infarct size on myocardial perfusion imaging not only provides information about the probable results of electrophysiological study, but can also give prognostic information after ICD implantation.

We found no significant difference in the extent of ischaemia on myocardial perfusion imaging between the inducible and the non-inducible patients. These results are in agreement with those of Gradel et al and Sellers et $a l .{ }^{19}{ }^{23}$ In the latter study, most of the patients with a history of VT and inducible tachycardia on electrophysiological study had fixed defects on 201-thallium perfusion scintigraphy. Only one third had some degree of ischaemia, and the morphological characteristics of the induced tachycardia and incidence of exercise induced VT showed no correlation with ischaemia. Although we previously showed that asymptomatic ischaemia on planar thallium images has prognostic significance for the recurrence of ventricular arrhythmias, ${ }^{24}$ Gioia et al recently showed that the presence or absence of ischaemia did not have a significant impact on survival in patients with life threatening arrhythmias treated with ICD. ${ }^{21}$ Further studies are necessary to evaluate the role of ischaemia in patients with documented life threatening ventricular arrhythmias.

\section{LIMITATIONS}

A major limitation of our study was the variability of QT measurements between and within observers. ${ }^{625}$ To minimise this problem, all calculations were done in this study by two independent experienced investigators who were blinded to the results of the electrophysiological study. Furthermore 20 patients (31\%) were excluded because the end of the $T$ wave could not be identified in at least eight leads. This is a particularly important problem when precordial repolarisation is abnormal-which is often the case in patients with documented myocardial infarction. A potential selection bias owing to exclusion of these patients is unlikely, as there were no significant differences in clinical characteristics and measurements of infarct size between the included and excluded patients. Furthermore, the interobserver variability for QT dispersion was within $11 \mathrm{~ms}$, which is lower than other studies in similar patient populations. ${ }^{26}$ Finally, it has been suggested that correction of the QT interval for the influence of heart rate using Bazett's formula is unreliable at low and high heart rates, as it is based on predominant heart rates in the studied population. ${ }^{27}$ However, the correction was used in all subgroups and the results were similar for corrected and uncorrected QT intervals.

\section{CONCLUSIONS}

There are no correlations between measurements of infarct size and QT parameters in patients with coronary artery disease and life threatening ventricular arrhythmias. In these patients, QT parameters do not predict inducibility during electrophysiological testing. In contrast, the amount of scar tissue, determined by myocardial perfusion imaging, is strongly correlated with inducibility.

We wish to thank Ludwig D'Hondt for his assistance during the electrophysiological studies and the data collection.

1 Van De Loo A, Arendts W, Hanloser S. Variability of QT dispersion measurements in the surface electrocardiogram in patients with acute myocardial infarction and in normal in patients with acute myocardial infarction

2 Zareba W, Moss A, le Cessie S. Dispersion of ventricular repolarization and arrhythmic cardiac death in coronary artery disease. Am f Cardiol 1994;74:550-3.

3 Barr C, Waas A, Freema M, et al. QT dispersion and sudden unexpected death in chronic heart failure. Lancet 1994;343: $327-9$.

4 Oikarinen L, Viitasalo M, Toivonen L. Dispersion of the QT interval in postmyocardial infarction patients presenting with ventricular tachycardia or with ventricular fibrillation. Am 7 Cardiol 1998;81:694-7.

5 Pye M, Quinn A, Cobbe A. QT interval dispersion: a non-invasive marker of susceptibility to arrhythmia in patients with sustained ventricular arrhythmia. Br Heart $f$ 1994;71:511-14.

6 Perkiömäki J, Koistimen M, Yli-Mäyry S, et al. Dispersion of QT interval in patients with and without susceptibility to ventricular tachyarrythmias after previous infarction. $f \mathrm{Am}$ Coll Cardiol 1995;26:174-9.

7 Lee K, Okin P, Kligfield P, et al. Precordial QT dispersion and inducible ventricular tachycardia. Am Heart $\mathcal{F}$ 1997; 134:1005-13

8 Sylver J, Horacek M, Spencer C, et al. QT interval variability on the body surface. F Electrocardiol 1984;17:179-88.

9 Day C, McComb J, Campbell R. QT dispersion in sinus beats and ventricular extrasystoles in normal hearts. $\mathrm{Br}$ Heart f 1992;67:39-41.

10 Garson A. How to measure the QT interval: what is normal? Am f Cardiol 1993;72:14-16B.

11 Bazett $\mathrm{H}$. An analysis of time relations of electrocardiograms. Heart 1920;7:353-70.

12 Wellens H, Brugada P, Stevenson W, et al. Programmed electrical stimulation of the heart in patients with life-threatening ventricular arrhythmias: what is the significance of induced arrhythmias and what is the correct cance of induced arrhythmias and what is
stimulation protocol? Circulation 1985;72:1-7.

13 Yumus A, Gillis A, Duff $\mathrm{H}$, et al. Increased precordial QTc dispersion predicts ventricular fibrillation during acute dispersion predicts ventricular fibrillation during
myocardial infarction. Am $\mathcal{f}$ Cardiol 1996;78:706-8.

14 Schneider C, Voth E, Bar F, et al. QT dispersion is determined by the extent of viable myocardium in patients with chronic Q-wave myocardial infarction. Circulation 1997;96:3913-20.

15 Weber K, Pick R, Silver M, et al. Fibrillar collagen and remodeling of dilated canine left ventricle. Circulation 1990;82:1387-401.

16 Dean J, Lab M. Arrhythmia in heart failure: role of mechanically induced changes in electrophysiology. Lancet 1989;333:1309-11.

17 Taggart P. Mechanico-electric feedback in human heart. Cardiovasc Res 1996;32:38-43.

18 Dobran I, Vester E, Strauer B. QT dispersion in patients with myocardial ischemia and good left ventricular function: is it suitable for risk stratification? [abstract] $7 \mathrm{Am}$ Coll Cardiol 1998;31(suppl C):53C.

19 Gradel C, Jain D, Batsford W, et al. Relationship of scar and ischemia to the results of programmed electrophysiological stimulation in patients with coronary artery disease. $7 \mathrm{Nucl}$ Cardiol 1997;4:379-86.

20 McFarland T, McCarthy D, Makkler P, et al. Relation between sites of origin of ventricular tachycardia and relative left ventricular perfusion and wall motion. Am $\mathcal{F}$ Cardiol 1983;51:1329-33.

21 Gioia G, Bagheri B, Gottlieb C, et al. Prediction of outcome of patients with life-threatening ventricular arrhythmias treated with automatic implantable cardioverterdefibrillators using SPECT perfusion imaging. Circulation 1997;95:390-4

22 De Sutter J, Tavernier R, Van de Wiele C, et al. The incidence of appropriate shocks in patients with coronary artery disease and life-threatening arrhythmias treated with implantable cardioverter defibrillators, is determined by the extent of scarring on perfusion imaging [abstract]. $f \mathrm{Am}$ Coll Cardiol 1998;31 (suppl C):58C. 
23 Sellers D, Beller G, Gibson R, et al. Prevalence of ischemia by quantitative Tl-201 scintigraphy in patients with ventricular tachycardia or fibrillation inducible by pro-

24 Jordaens L, Hollanders G, De Schrijver A, et al. Incidence Jordaens L, Hollanders G, De Schrijver A, et al. Incidence
and prognostic significance of asymptomatic ischaemia in patients with sustained ventricular arrhythmias. Eur Heart $\mathcal{F}$ 1988;9(suppl N):128-35.
25 Savelieva I, Yi G, Guo X, et al. Agreement and reproducibility of automatic versus manual measurement of QT interval and QT dispersion. Am $f$ Cardiol 1998;81:471-7.

26 Glancy J, Garrat C, Woods K, et al. QT dispersion and mortality after myocardial infarction. Lancet 1995;345:945-8. 27 Ward D. Prolongation of the QT interval as an indicator of risk of a cardiac event. Eur Heart $\mathcal{F}$ 1988;9:139-44.

\section{IMAGES IN CARDIOLOGY}

\section{Patent foramen ovale}
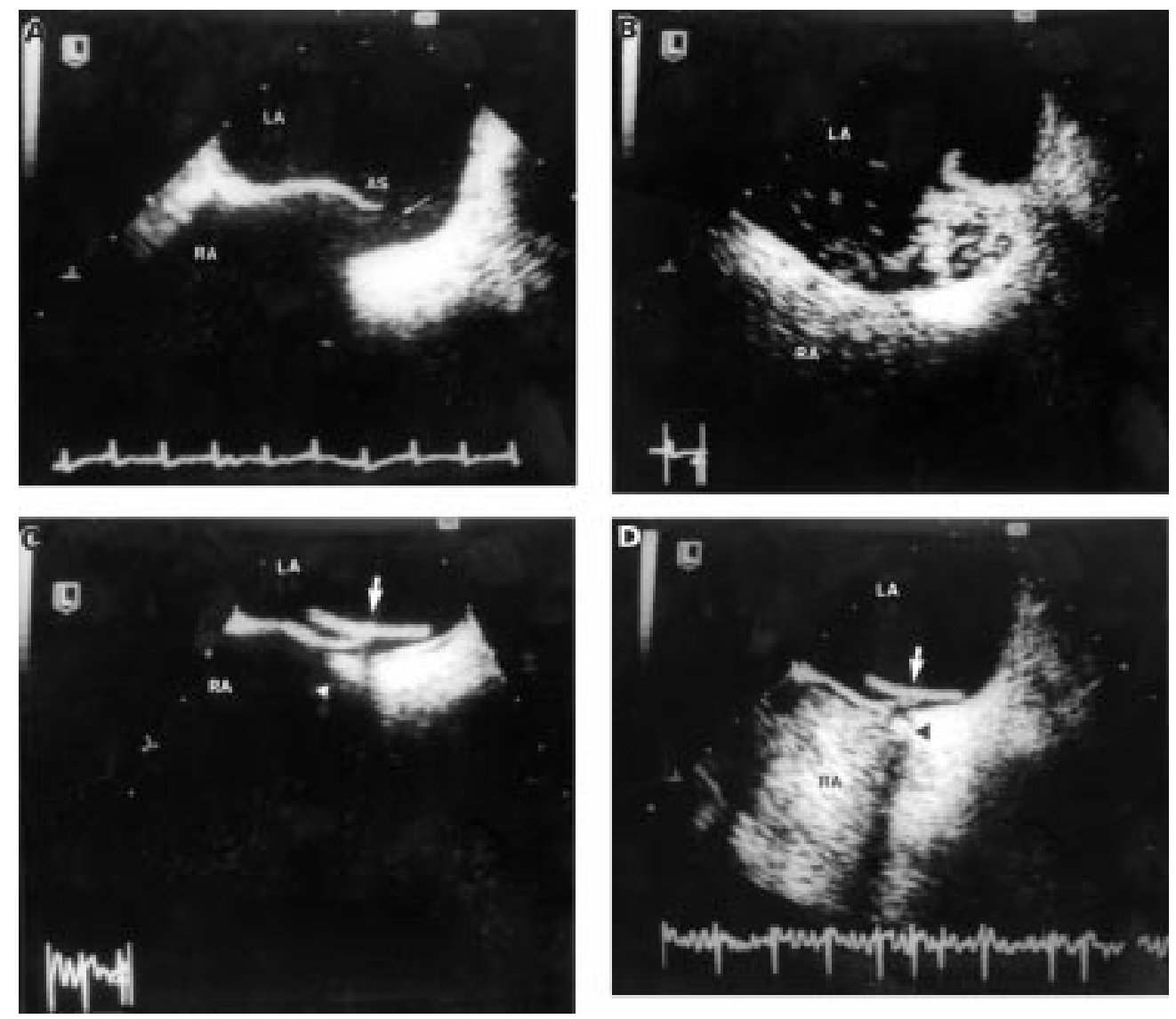

A 61 year old man presented with a cryptogenic cerebrovascular insult. Transoesophageal echocardiography (A) showed a patent foramen ovale (thin arrow). Agitated Haemaccel (polygeline, Hoechst Marrion Roussel, Middlesex, UK) was injected intravenously as a $2 \mathrm{ml}$ bolus and flushed with $10 \mathrm{ml}$ of saline $0.9 \%$. During Valsalva manoeuvre, a right to left shunt was demonstrated through the patent foramen ovale of the interatrial septum (B). A buttoned occluder was placed successfully

during cardiac catheterisation. Echocardiography was not used during the procedures. Transoesophageal echocardiography the next day demonstrated a good position of the occluder (arrow) and counter occluder (arrowhead) (C) without and (D) with right atrial contrast filling. (AS, atrial septum; LA, left atrium; RA, right atrium.)

TUSHAR CHATTERJEE
BEAT AESCHBACHER BERNHARD MEIER 